\title{
On the possibility of getting traceable time and frequency measurements via GNSS receivers
}

\author{
Andreas Bauch $^{1}\left(\mathbb{0} \cdot\right.$ Dirk Piester $^{1}\left[\right.$ ] Thomas Polewka $^{1} \cdot$ Egle Staliuniene $^{1}$
}

Received: 3 July 2020 / Accepted: 10 August 2020

(c) The Author(s) 2020, corrected publication 2021

\begin{abstract}
The realization of Coordinated Universal Time, one of the tasks of the International Bureau of Weights and Measures, relies on a network of international time links which currently is organized in a star-like scheme that links all contributing laboratories. GPS signal reception is the technique most widely employed by the laboratories. The PTB currently plays a unique role in the process due to its function as the central pivot in the time transfer between the participating laboratories. We discuss how the PTB meets its obligations to the international timekeeping community as well as to its users in Germany. In its role as an National Metrology Institute (NMI), PTB is entrusted with the realization and dissemination of legal time in Germany. The services were offered to the public support measurements and timing applications traceable to the national and international standards to be made in calibration laboratories and in many industrial sectors. We thus discuss the meaning and definition of traceability, how different GNSS systems can be used to establish traceability and their performance in doing so.
\end{abstract}

Keywords GNSS · GPS · Galileo · Time and frequency metrology · Traceability

\section{Introduction}

Assured access to accurate time has been identified as indispensable for the functioning of modern infrastructure worldwide. In virtually all sectors, namely energy, telecommunications, finance, and-quite naturally-positioning and navigation, but also time and frequency metrology, the reception of signals from Global Navigation Satellite Systems (GNSS) has found widest use. The worldwide collaboration in realizing Coordinated Universal Time (UTC) as the commonly agreed time reference was recently described by Panfilo and Arias (2019) from the International Bureau of Weights and Measures (BIPM). It relies to a large extent

Disclaimer Commercial Products are identified for the sake of technical clarity. No endorsement by the authors or their institute is implied.

This article is published as part of the special collection

"Timekeeping in space: technology, practice, promise, and benefits".

Andreas Bauch

Andreas.bauch@ptb.de

1 Physikalisch-Technische Bundesanstalt (PTB), Bundesallee 100, 38116 Braunschweig, Germany on time comparisons involving signal reception from the US Global Positioning System (GPS). Each of the about 80 timing institutes worldwide operates one or more specialized GNSS timing receivers, which relate the time of arrival of GNSS signals to the local time scale for this purpose. Several laboratories perform Two-Way Satellite Time and Frequency Transfer through geostationary satellites in parallel. Through such time transfer links, the timing institutes get access worldwide get access to the realization of the SI unit of time, the second, and to time marks in the agreed common time reference UTC, even without operating sophisticated high-accuracy atomic clocks. GPS remains the single source for many institutes. For quite some years, the Physikalisch-Technische Bundesanstalt (PTB), Germany's National Metrology Institute (NMI), serves as the pivot for the time links as it is equipped with all kinds of time transfer equipment, including redundant GNSS receivers. For almost 20 years, PTB has also been a site in the network of GNSS observation stations of the International GNSS Service (IGS) (Johnston et al. 2017). The station has been overseen by the German Bundesamt für Kartographie und Geodäsie (BKG) during all the years. The installations maintained at PTB and their performance are described in the next section. In order to make accurate time comparisons with specified uncertainty feasible, the signal delays in the receivers are 
characterized as will be described in some detail. PTB-as other timing institutes-has continued to support BIPM in this undertaking using traveling equipment that is shipped around in Europe and occasionally elsewhere. As a result, the delays for GPS signals received at the two legacy frequencies L1 and L 2 can be determined with a $1-\sigma$ uncertainty of about 1 to $1.5 \mathrm{~ns}$.

By far, the largest number of GNSS receivers operated worldwide for timing applications is used in so-called GNSS-disciplined oscillator devices that provide standard frequency and time signals or are embedded in servers providing time information via IT protocols like NTP or PTP. There is an ongoing debate on how traceability to national or international standards can be established with reasonable effort using such instrumentation, as reflected in the title of this paper. PTB shares common consensus that "something has to be done" (Lombardi 2016; Matsakis et al. 2018; Piester et al. 2019a), and in the section on recording and documentation of GNSS signals in PTB, our approach is provided for discussion. Looking ahead, improvements in the quality of time comparisons are needed to keep up with the ongoing improvements of atomic clocks. One way could be to use another GNSS in addition to GPS, and illustration of the first steps is given.

\section{GNSS time transfer for the realization of UTC}

The comparison of clocks operated in timing institutes worldwide has always been an important part of time metrology. Reception of GPS satellite signals started to be used for the purpose in the early 1980s (Allan and Weiss 1980). A compilation of various aspects of satellite time and frequency transfer can be found in a Handbook of the International Telecommunications Union, Radiocommunication Sector (ITU 2010), and the current status has also been sketched in the above-mentioned recent BIPM publication (Panfilo and Arias 2019).

\section{General description}

In brief, at the time of writing, the BIPM collects GNSS observation data (and, as far as available, data from other time transfer techniques) from about 80 laboratories marked as colored dots on the World map in Fig. 1 that form a nonredundant network of comparisons with PTB as the node of this scheme. Each of the laboratories, designated as " $k$," generates a real-time physical realization of UTC, named $\mathrm{UTC}(\mathrm{k})$, which is compared to UTC(PTB). Seventy-three time links between timing laboratories and PTB are routinely evaluated using only GPS reception data as follows from the recently issued Circular T 387, April 2020. The Circular $\mathrm{T}$ is a monthly publication of the BIPM that provides the differences between UTC and UTC(k) for the regularly contributing institutes. Fifteen institutes still provide only single-frequency (GPS L1) data from a multi-channel receiver [GPS MC], whereas the others provide dual-frequency data. The latter can be processed as the ionosphere-free linear combination in either code-based manner [GPS P3] or combined with carrier-phase observations [GPSPPP], depending on the availability of appropriate measurement data. The designations in square brackets are those used in Circular $\mathrm{T}$, Section 5 , to identify the computation procedure. Codebased data are reported in the so-called CGGTTS format (Defraigne and Petit 2015), whereas carrier-phase observations are reported in RINEX files (Johnston et al. 2017; IGS 2015).

\section{Characterization of PTB's GNSS receivers}

PTB provides GPS observation data in the formats of RINEX and CGGTTS V2E from four receivers to BIPM, complemented by BeiDou, Galileo and GLONASS data that are not yet routinely used. The PTB "golden" reference receiver used to be a legacy Ashtech Z-12T with the designation PTBB (IGS station providing RINEX data) and PT02 (for CGGTTS data) up to April 2019. A long-term record of local common-clock common-view comparison between PT02 and two other receivers is shown in Fig. 2. Because the internal signal delays of the stations had been calibrated before, one would ideally expect in such a setup measurement noise centered around zero mean value. The apparent seasonal variation was likely caused by delay variations in the PT02 antenna cable that was laid as early as 2002.

In April 2019, BKG replaced the old GPS-only receiver with a Septentrio PolaRx5TR receiver, which can track all GNSS, in particular Galileo and GLONASS. The designation of the IGS station (PTBB) was kept, but the designation for CGGTTS data was changed to PT13. Since July 2019, all links to PTB are established through PT13 by BIPM after a few months of use of receiver PT09, which is a Septentrio PolaRx4, according to Circular T 379, August 2019. PTBB is also part of the EUREF Permanent Network (EPN) of observation stations for which the tracking performance is documented (Bruyninx et al. 2019; EUREF 2020). For illustration, in Fig. 3, the code multipath statistics for the GPS L1 frequency observations and the number of identified phase cycle slips of station PTBB are shown. The EPN Central Bureau (EPN CB) also provides a comparison of individual performance against the large number of stations in the EPN. Here, one still notes some deficiencies of PTBB, which can probably be explained by the local conditions for mounting the antenna. Not all the guidelines for antenna installation published by the IGS (IGS 2013) can be met on the PTB campus. In Fig. 4, a view of the roof installation of several GNSS antennas is presented. Comparing PT13 with other 


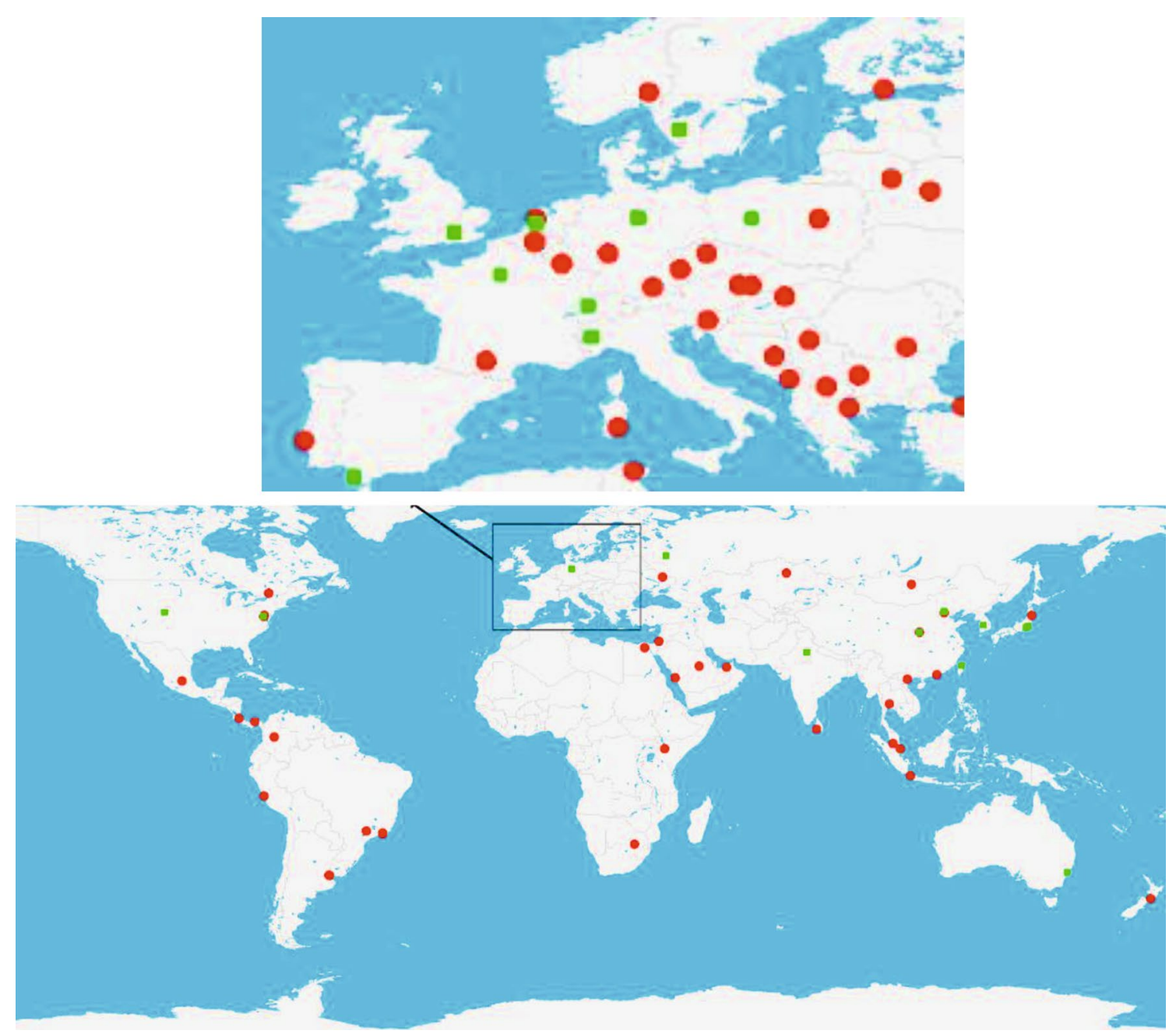

Fig. 1 Geographical distribution of the laboratories that contribute to UTC, European laboratories in the upper panel (source: BIPM, reproduced with kind permission of BIPM). Institutes marked with green squares operate ground stations for Two-Way Satellite Time and Frequency Transfer in addition to their GNSS receivers
PTB receivers during the first year after installation reveals consistency among all data that are provided to BIPM, and temporal variations are bound to within $0.5 \mathrm{~ns}$ as illustrated in Fig. 5. The offsets are discussed in the following section.

\section{Determination of GNSS signal delays in receivers}

The quantity plotted in Figs. 2 and 5 is the difference between daily mean values of UTC(PTB) and GPS time, which is based on typically 700 observations of 16-min duration recorded in a pair of receivers. In addition to the geometrical propagation delay between satellite and receiving antenna, the individual results are affected by the signal delay in the antenna cable [CAB DLY], the time offset between the UTC(PTB) reference point and the receiver internal measurement point [REF DLY] and the signal propagation delay through the antenna and inside the receiver up to the measurement point [INT DLY]. Designations in square brackets are defined in the CGGTTS (Defraigne and Petit 2015). Whereas the first two delay types are common for all received signals and can be determined by the operator, the internal delay is known to be noticeably code and frequency dependent and more difficult to obtain. The characterization of these delays is generally termed as "receiver calibration," and it is essential to the accuracy of time transfer and time dissemination. The complete set of GNSS equipment in laboratories used for time transfer in the context of UTC generation thus needs to be calibrated. This activity has been organized by defining two groups of laboratories (BIPM 2016): Each regional metrology organization selected one or a few so-called group 1 (G1) laboratories. The BIPM conducts campaigns for the determination of the INT DLY of their receiver(s). The G1 laboratories, in turn, conduct campaigns of the same kind for the larger number of 


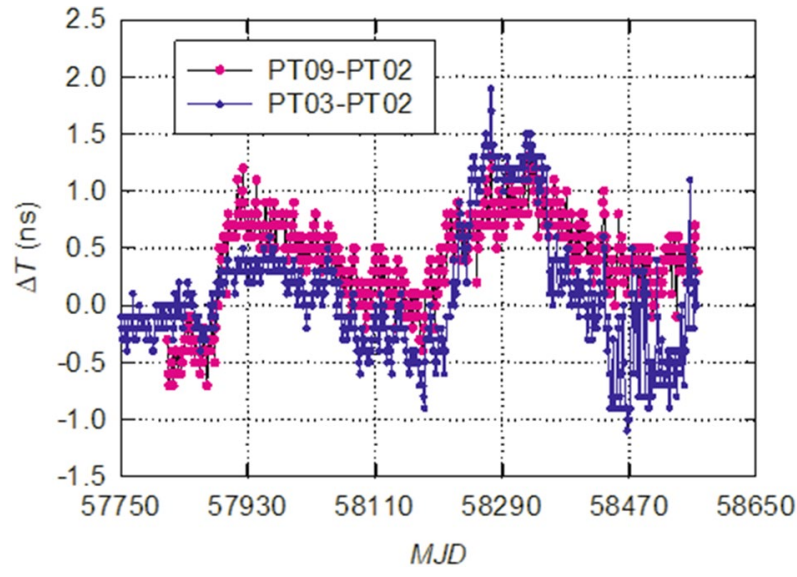

Fig. 2 Two and a half years of daily mean values of common-clock common-view comparison (GPS, ionosphere-free) between receivers PT03 of type Ashtech Z-12T and PT09 of type Septentrio PolaRx4 with the previous PTB reference receiver PT02 (Ashtech Z-12T). The Modified Julian Date (MJD) 57750 corresponds to December 28, 2016

so-called G2 laboratories in their region. Their total number in Europe is 31, with some 70 GNSS receivers in operation. Ideally, such calibrations should be repeated every few years, but this has almost never been achieved.

The current BIPM GNSS calibration guidelines (BIPM 2016) were first released in 2013, but such calibrations have happened more or less frequently since the 1990s. Historically, the receiver delays of BIPM equipment (up to today) have been based on the so-called absolute calibration in which synthetic, simulated signals were employed at the US Naval Research Laboratory (White et al. 2001). In brief, in such absolute calibration, the signal delays in all components of the receiving chain (antenna, receiver and cable) are each measured individually using simulated signals produced by signal generators. The "ideal" signals produced in a GNSS signal simulator may deviate from the signals received from the spacecraft, a fact that was more recently discussed in the context of the use of Galileo (see below). Since 2014, the BIPM G1 reference receiver delays were adjusted to provide a continuation to the values of three historical receivers. Nowadays, the reference value for each new G1 campaign is defined by an ensemble of receivers that took part in the preceding campaign (see https://www.bipm.org/jsp/en/TimeC alibrations.jsp, Calibration Identifier 1001-2018).

PTB has often hosted BIPM traveling receivers. This was important as any changes to the "golden receiver" would have affected all links. Although this is more of academic interest today, Table 1 contains the results of the INT DLY for the ionosphere-free dual-frequency linear combination, designated by P3 as determined over the years for receiver PT02. This quantity was relevant for all links of type [GPS P3] and [GPSPPP], respectively, up to April 2019. The peak-to-peak variation did not exceed $2 \mathrm{~ns}$, which complies well with the $1-\sigma$ uncertainty of between $1 \mathrm{~ns}$ and $1.5 \mathrm{~ns}$ for each individual value. There was no overlap in operations between PT02 and the new PT13 as the same monument was to be used for mounting the new antenna. The GPS signal delays in PT13 have thus been determined based on the PT09 results reported for the BIPM campaign referred to in the previous paragraph.

In cooperation with BIPM and alike French and Spanish institutes, PTB continues to offer calibrations of GPS receivers to other (mostly) European G2 laboratories. Between 2018 and 2020, PTB has organized and conducted the socalled G1G2 calibration trips to FMTC (Vilnius, Lithuania), GUM and AOS (Warsaw and Borowiec, Poland), NMISA (Pretoria, South Africa), IMBIH (Sarajevo, Bosnia and Herzegovina), DTAG (Frankfurt am Main, Germany), NIMB (Bucharest, Romania) and SIQ (Ljubljana, Slovenia). Results of all campaigns are published on the BIPM Web as referred to above, and the detailed calibration reports are available for download. The traveling receiver used hitherto has been a dual-frequency GPS (only) receiver of type MESIT GTR50 (Feldmann et al. 2010). In November 2019, the assembly of a new traveling receiver kit, including a Septentrio PolaRx5TR receiver and a small form-factor time interval counter, was finished. Subsequently, receiver delays at VTT (Finland) were successfully determined. The new setup shown in Fig. 6 should be simpler to transport and operate than the older, rather bulky device. The transfer of calibration results from one to another receiver locally is straightforward. It is, however, only possible with a nonnegligible uncertainty, below $1 \mathrm{~ns}$ in the most favorite cases. This explains the offsets seen in Fig. 5 among the various receivers.

In summary of this section, we briefly discuss the uncertainties for the differences between UTC and UTC $(k)$ reported in the BIPM Circular $\mathrm{T}$ for laboratories " $\mathrm{k}$ " that are linked via GPS only. The report of the time differences, including their uncertainty, is documentation of the participation of the NMI in the BIPM Key Comparison CCTFK001.UTC, which is the agreed method for establishing traceability to the unit of time, the second, in the International System of Units (SI) and its inverse, $\mathrm{s}^{-1}=\mathrm{Hz}$ for frequency. Participation to the Key Comparison is a priori strictly limited to NMIs which maintain an approved quality system and are signatories of the Mutual Recognition Arrangement, drawn up by the International Committee of Weights and Measures under the authority given to it in the Metre Convention. NMIs can designate institutes (DI) in their respective country to fulfill their tasks in certain metrology fields. The BIPM Circular T, however, reports the results also for the about ten laboratories that are engaged in time and frequency metrology for their internal scientific purposes. This situation may cause some confusion but 

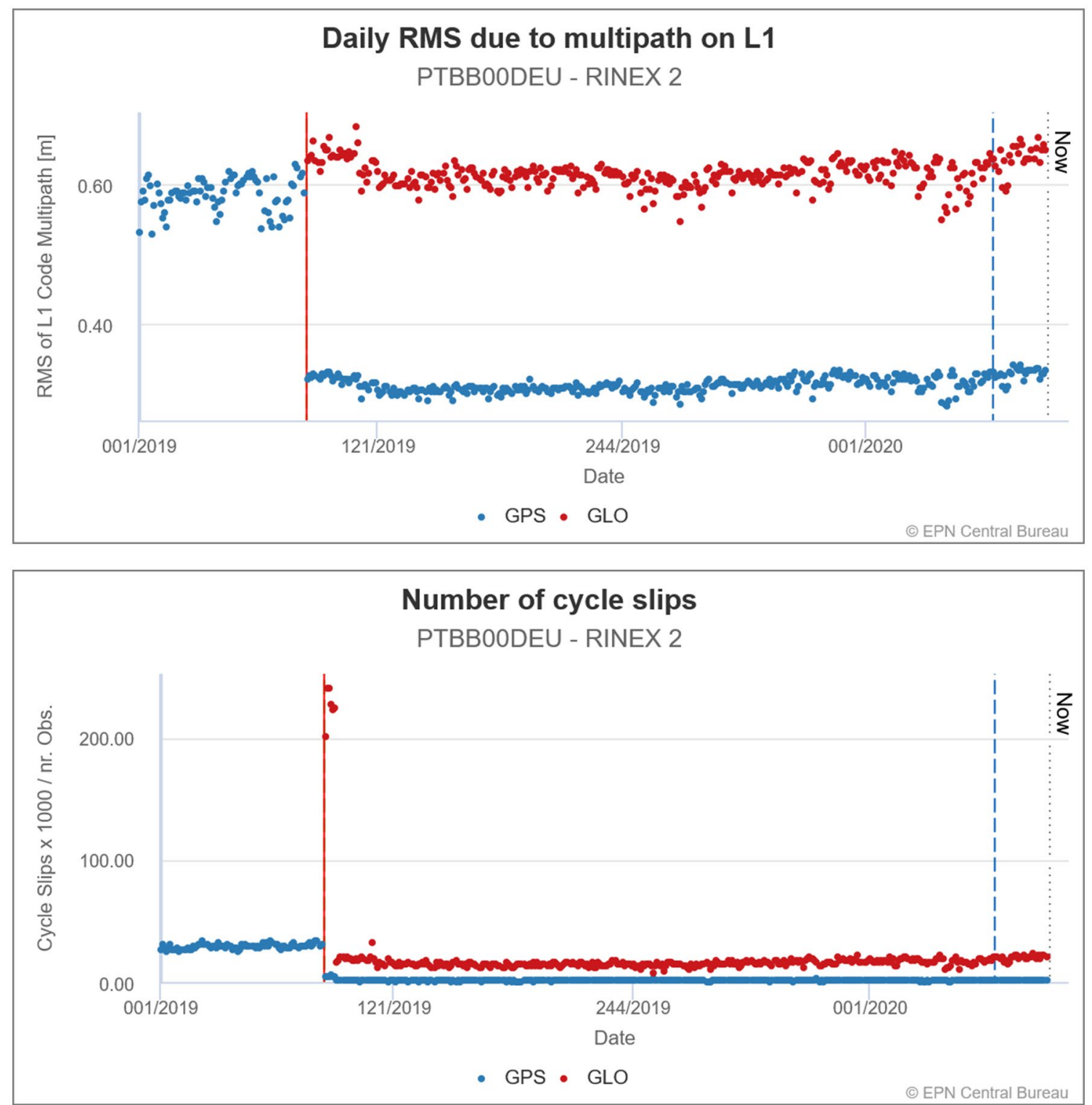

Fig. 3 Changes in tracking the performance of station PTBB as documented by EPN CB (EUREF 2020). The red vertical lines indicate the change of receiver and antenna, and the dashed blue vertical lines indicate a firmware upgrade. Multipath suppression is activated in the new type of receiver and is noticeable in the upper panel. The reduc-

cannot be discussed further here. Based on the uncertainty for individual receiver calibrations, a conventional (minimum) value of $2.5 \mathrm{~ns}$ is assigned for each GPS-based link. The value is increased with time passed since a calibration has happened (Jiang et al. 2011) so that it today exceeds $3.0 \mathrm{~ns}$ when the last calibration is from 2016 or earlier at the time of writing. This is done irrespective of the equipment involved and without full transparency as to how to justify the rate of increase. The intention is, of course, understandable: Laboratories shall be encouraged to maintain an tion in the number of cycle slips, visible in the lower panel, will facilitate long-term carrier-phase data analysis. Both plots run from day 1, 2019, to day 93, 2020. Reproduction with kind permission of EPN $\mathrm{CB}$, Observatoire Royale de Belgique

up-to-date calibration of their local installations constantly. During its meeting in June 2020, the CCTF Working Group on GNSS decided that the pressure on the laboratories to repeat calibrations frequently should be slightly relaxed as the stability of most receivers has been found good. An uncertainty of $20 \mathrm{~ns}$ is assigned to those laboratories which are somewhat negligent in this direction, and their number amounts to 17 in the April 2020 issue of the Circular T. The value of $20 \mathrm{~ns}$ has been chosen in 2005 by BIPM in order to distinguish uncalibrated laboratories clearly. On the other 


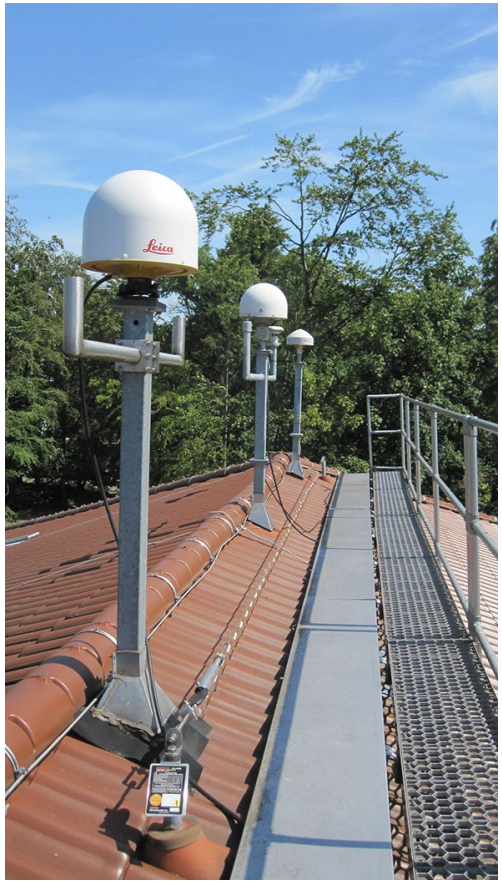

Fig. 4 Installation of GNSS antennas on the rooftop of PTB's clock hall. The direction of view is toward the northeast. Starting from the new PTBB antenna in the foreground, we see toward the background the antennas of receivers PT09, PT10 of type MESIT GTR51 and GRCP of type MESIT GTR55, respectively

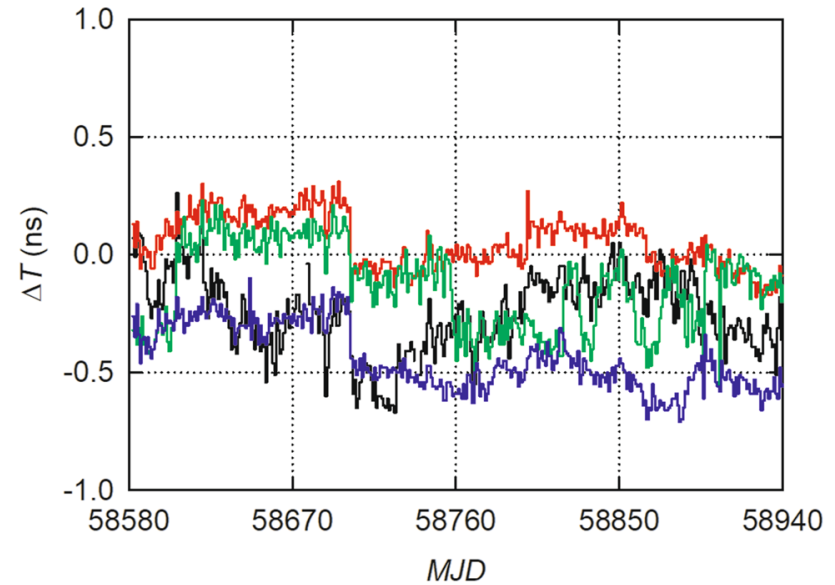

Fig. 5 Daily mean values of common-clock common-view comparison (GPS ionosphere-free) between various receivers with PT13 for almost 1 year; black: GRCP (GTR55), red: PT09 (PolaRx4), green: PT10 (GTR51) and blue: PT11 (PolaRx4). The last data point is from March 25, 2020. A systematic trend can hardly be detected in the data, and the maximum linear slope observed (for PT11-PT13) is below $1 \mathrm{ps} / \mathrm{d}$

hand, the present algorithm for evaluating the uncertainties of UTC-UTC(k) (Panfilo and Arias 2019) requires that a not too large value is chosen for an uncalibrated laboratory. As we will discuss in the following section, calibration of signal
Table 1 Results of PT02 delay determinations between 2008 and 2018

\begin{tabular}{lll}
\hline Year & Source (report code) & INT DLY (P3) (ns) \\
\hline 2008 & BIPM (1001-2008) & 281.33 \\
2012 & Unpublished & 282.32 \\
2014 & BIPM (1001-2014) & 282.34 \\
2014 & OP & 282.75 \\
2015 & NIST/OP/PTB & 282.2 \\
2016 & OP & 282.5 \\
2016 & BIPM (1001-2016) & 280.9 \\
2018 & BIPM (1001-2018) & 282.1 \\
\hline
\end{tabular}

Since 2014, campaigns have been identified uniquely (BIPM 2016). Colleagues from the French NMI LNE SYRTE working at the Observatoire de Paris (OP) conducted two campaigns designated "OP" in the frame of the operation of the Galileo Time Validation Facility. The 2015 results came from a joint NIST/OP/PTB campaign whose complete results have not been published. Here, NIST stands for National Institute of Standards and Technology, the US NMI

delays is not of prime concern for laboratories that wish to obtain traceability for the time unit or for frequency only, and not for time (the epoch) in UTC.

\section{Recording and documentation of GNSS signals in PTB}

We have discussed how the use of GPS in the international collaboration of timing laboratories and NMIs has been successfully established and gives them traceable access to the SI unit of time. But many measurements are being made in the industry, in calibration facilities and in the context of telecommunications, energy supply and in the financial sector. It is the NMI's specific task, entrusted to them by their respective national authority, to make available to their country an efficient metrological infrastructure recognized on the international level. This would enable traceable measurements and time stamping to be made, and we explain next in more detail the concept of metrological traceability.

\section{Discussion of traceability}

In clause 2.41 of the Vocabulaire international de métrologie (VIM) (JCGM 2012), metrological traceability is defined as "property of a measurement result whereby the result can be related to a reference through a documented unbroken chain of calibrations, each contributing to the measurement uncertainty." The term "calibrations" in the preceding phrase has a wider meaning than what we referred to earlier. In a previous edition of the VIM, the term in use was instead "comparison" which would be easier to understand. From our point of view, the keywords are documented 
Fig. 6 New PTB traveling GNSS receiver used for

G1G2 calibrations. The setup comprises a receiver and a timeinterval counter mounted in a 19 " rack mount chassis, shown on the photograph, a $65 \mathrm{~m}$ antenna cable and a NavXperience $3 \mathrm{G}+\mathrm{C}$ GNSS antenna

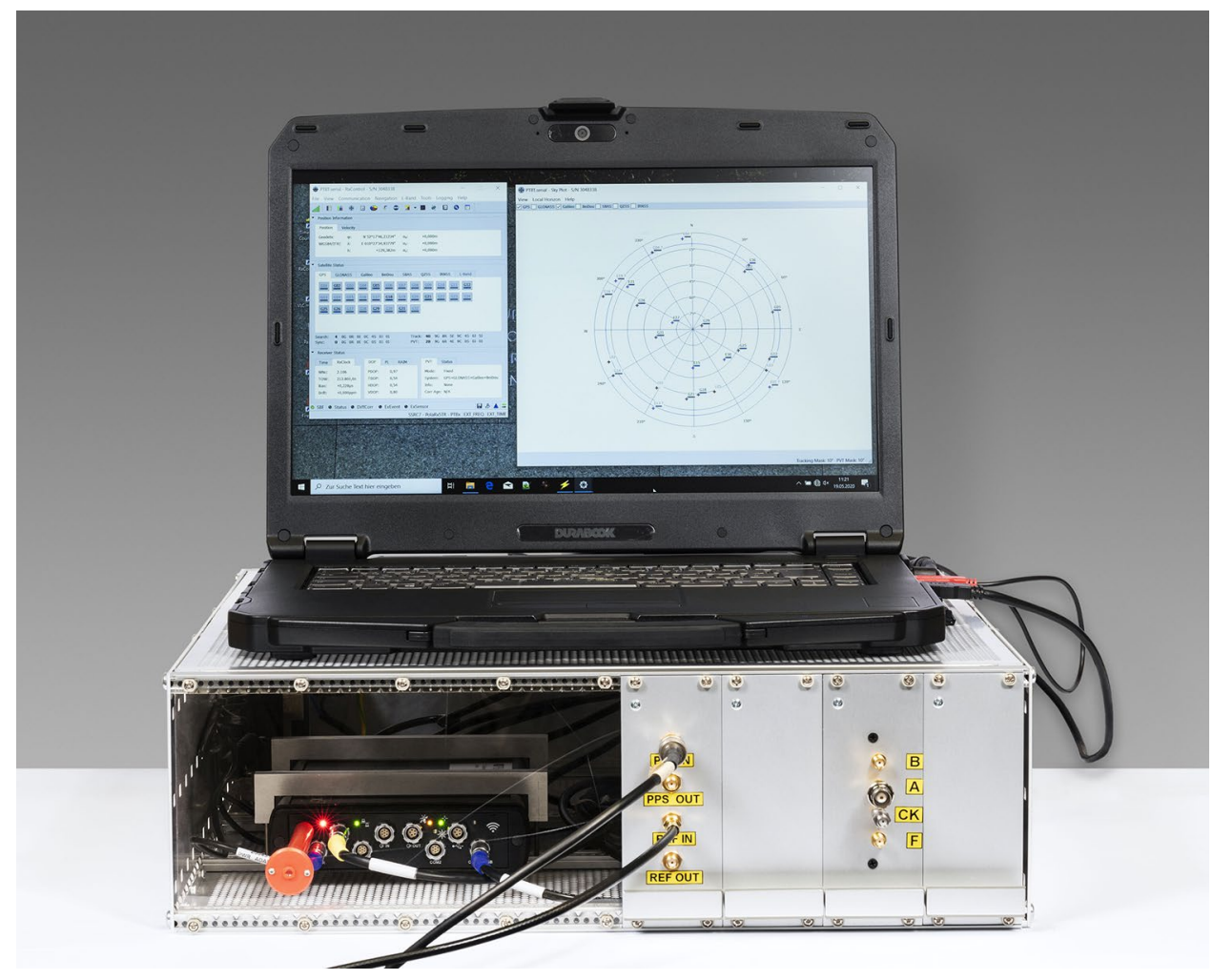

and measurement uncertainty. Requirements for traceability may be imposed from national accreditation bodies or can even be stated in a law such as the German Telecommunications Act. The operator thus needs to satisfactorily show an uninterrupted traceability chain from his measurement setup to the primary frequency and time reference. One of the UTC(k) timescales realized by an NMI or DI is such a suitable reference. Many calibration laboratories and IP-based time distribution in local area networks, however, employ GPS-disciplined oscillators (GPSDO) as their reference. GLONASS may be attractive in some regions, and Galileo is just being introduced in the market for such devices. Subsequently, we discuss to which extent such economically and technically favorable practice is formally justified. The technical function and issues of GPSDOs have been treated well in the literature (Lombardi 2016; Piester et al. 2019a), and a guide for accredited calibration laboratories in Europe is available (EURAMET 2016) so that we need not repeat this here.

\section{Using GPS signals as time and frequency reference}

"GPS time is established by the Control Segment and is referenced to Coordinated Universal Time (UTC) as maintained by the US Naval Observatory [UTC(USNO)] zero time-point defined as midnight of January 5/January 6, 1980. The OCS shall control the GPS time scale to be within one microsecond of UTC (modulo one second)." This quote from the GPS Interface Specification (GPS 2019) defines the starting epoch of GPS time when it agreed with UTC within a small fraction of a second. As leap seconds are not introduced in GPS time, it is in April 2020 ahead of UTC by 18 s. This number and a prediction of the offset between UTC(USNO) and GPS time are transmitted in the navigation message as explained in Section \$20.3.3.5.2.4 of GPS (2019). In the recent GPS Standard Positioning Service Performance Standard [GPS SPSPS 2020], the accuracy of the transmitted offset, called UTC Offset Error, is specified in Table 3.4-4 as $<30$ ns (95\% confidence level). In Fig. 7, this daily prediction is contrasted with the differences between UTC and UTC(USNO) and UTC and UTC(PTB). During recent years, the three timescales have deviated by less than $5 \mathrm{~ns}$ so that the information in the navigation message can de facto be considered as a good prediction for all of them. By US legislation, the reception of GPS establishes the required legal traceability path (Matsakis et al. 2018). In their paper, the authors also advertise services provided by NIST, for establishing a link to UTC(NIST). This service builds on GPS common-view comparison between the user and NIST and would be beneficial worldwide. The PTB activities explained below offer interested partners the opportunity to do the same kind of evaluation in their own responsibility. 


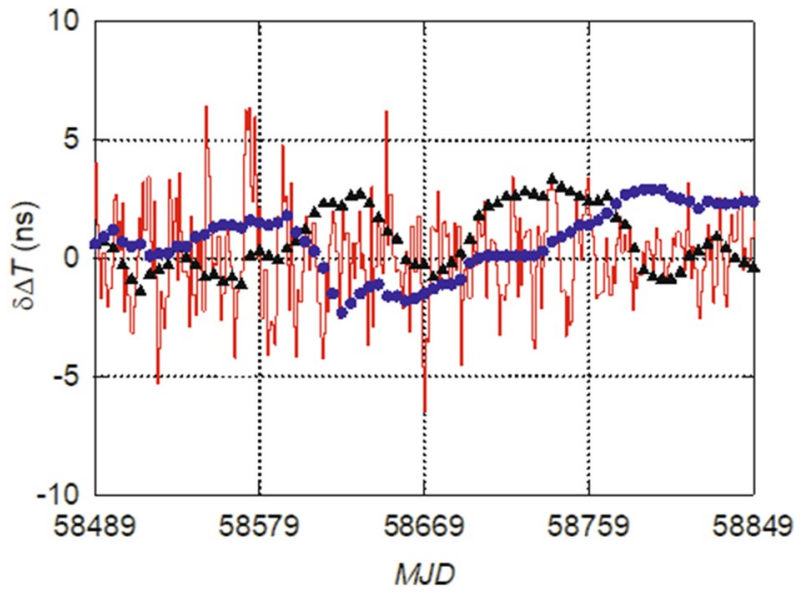

Fig. 7 Comparison of the predicted values of GPS timeUTC(USNO), red lines, daily values, and the differences UTCUTC(USNO) (black) and UTC-UTC(PTB) (blue), respectively, as reported in the BIPM Circular T during nearly 1 year, ending with January 1, 2020 (MJD 58849)

\section{Using Galileo signals as time and frequency reference}

The European GNSS Galileo is almost completed and ready for use. Initial services were officially declared feasible by the European Commission in December 2016. Public Galileo Programme reference documents are provided by the European GNSS Service Centre (URL: http://www.gsceu ropa.eu). Among other things, the Galileo Open Service Service Definition Document (OS SDD) contains the OS minimum performance level to be expected during this initial phase. Galileo System Time (GST) is realized in the ground segment as a physical signal, and it is the Time Service Provider who is responsible for its realization and alignment to UTC. GST follows GPS in the week numbering and in the format of reporting the predicted offset between GST and a prediction of UTC. Publications from an earlier phase of the development of Galileo describe the architecture and achieved performances (Píriz et al. 2015a, Galluzzo et al. 2018) in some detail. In the meantime, however, contractual responsibilities and technical implementation have significantly changed.

As stated before, accurate time comparisons require the signal delays to be calibrated. The delay for the Galileo E5a signals can be determined as described by Defraigne et al. (2014) based on an existing calibration of GPS signal delays. It is assumed that the Galileo E1 and GPS L1 signals share the same delay, and the Galileo Broadcast Group Delay (BGD) values need to be known. With the support of GMV, Tres Cantos, Spain, the internal E5a delays for several receivers operated in PTB were determined (Píriz et al. 2015b), and this process was repeated when new BGD values were released. The uncertainty for the delay values was stated as $3.7 \mathrm{~ns}$ for the E5a delay and $4.2 \mathrm{~ns}$ for the ionosphere-free linear combination (Defraigne et al. 2014). In 2018, receivers that had a so-called absolute calibration of Galileo delays were compared in common-clock mode to receivers that had the more traditional relative calibration (Garbin et al. 2019). The differences are of order of 2 ns. The Galileo delays in all PTB data were based on the results obtained in the relative mode up to early July 2020. The CCTF working group on GNSS, at its meeting held June 2020, decided that the Galileo reference for G1 calibrations would be realized through the absolute calibration of the BIPM receiver BP21 performed at ESTEC (Garbin et al. 2019). Subsequently, the BIPM published Galileo delay values for the $\mathrm{G} 1$ laboratories, including for PTB receiver PT09. Subsequently, the Galileo delay values of all PTB receivers were aligned to those of PT09.

Studies of international time comparisons using Galileo signals in comparison with GPS were made by Petit and Harmegnies (2019), demonstrating the advantages of using Galileo signals. Thus, the use of Galileo signals will be very attractive in GNSS-disciplined oscillators. Evidence for that comes from the reduced scattering of measurement data during a day, which is demonstrated in Fig. 8. On those days in 2018, the two GNSS timescales had a significant offset, which is visible in the figures. The improvement reflects a more accurate prediction of the satellite orbits in the navigation message. The so-called NeQuick model has been implemented in the Galileo system (European Commission 2015), which provides improved modeling of the ionosphere compared to the Klobuchar model implemented in GPS. This is advantageous for single-frequency users (Píriz et al. 2016) and is demonstrated in Fig. 9.

In the Galileo navigation message, the offset between GST and a prediction of UTC is reported. We have a doubt that the current documentation of GST realization and of the prediction algorithm could be accepted to provide traceability as discussed before. For the current initial operations phase, the OS SDD states that the prediction should be correct within $31 \mathrm{~ns}$. To our observation, this performance was always met. Recently, a significant mismatch between prediction and values recorded in PTB was noted and is illustrated in Fig. 10. It appeared and went away without any notice about the causes and final settling.

\section{PTB documentation}

GNSS monitoring bulletins published by some NMIs can provide a readily available means of confirming that the broadcast GNSS timing signals were correct at a given point in time. The bulletins support traceability between measurements made using the space signals as received and processed in a GNSS-disciplined oscillator and the UTC $(\mathrm{k})$ 

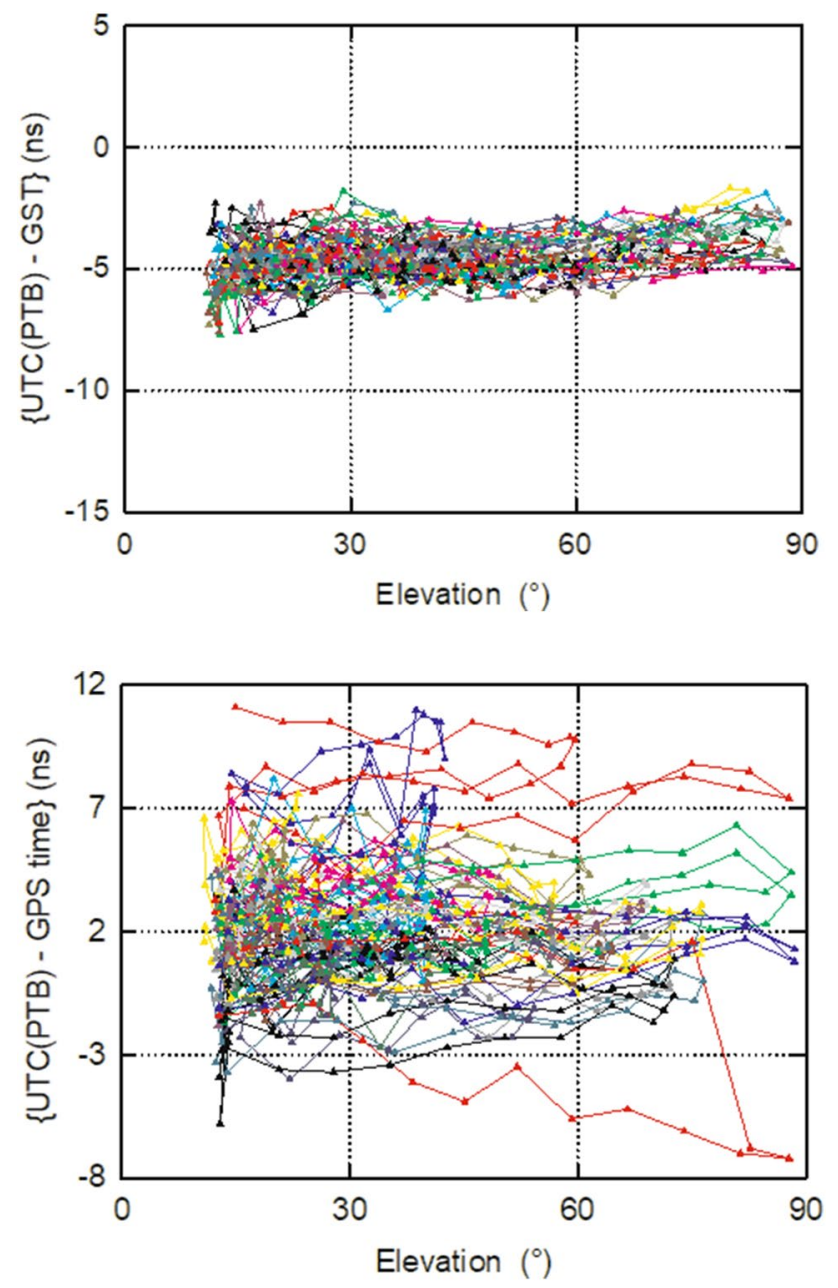

Fig. 8 Reception of Galileo and GPS signals in PTB as a function of elevation using a Septentrio PolaRx4 receiver with a Novatel 750.R4 antenna; Galileo (upper panel), GPS (lower panel, 20 satellites only). The quantity plotted is UTC(PTB) - GNSS time, on November 27 and 28, 2018. Data points connected by lines are from an individual satellite

timescale of the issuing NMI. In PTB, up to eight GNSS receivers from four different makers have been operated during recent years, and their observations are compared daily, as previously illustrated in Figs. 2 and 5. Observation files in CGGTTS format (Defraigne and Petit 2015) and-as far as possible-in RINEX format (IGS 2015) are publicly available for the previous day at ftp://ftp.ptb.de/pub/time/ GNSS/ in various folders. For the experienced user, these files provide a direct link between UTC(PTB) and GNSS timescales. For the general public, a weekly Time Service Bulletin (TSB) is published at ftp://ftp.ptb.de/pub/time/bulle tin/. Users in Germany who seek to obtain traceability to German legal time from GNSS signals are advised to take note of the contents of the TSB. A brief discussion of an uncertainty estimation can be found in Piester et al. (2019b). We have also given guidance on how data analysis should be
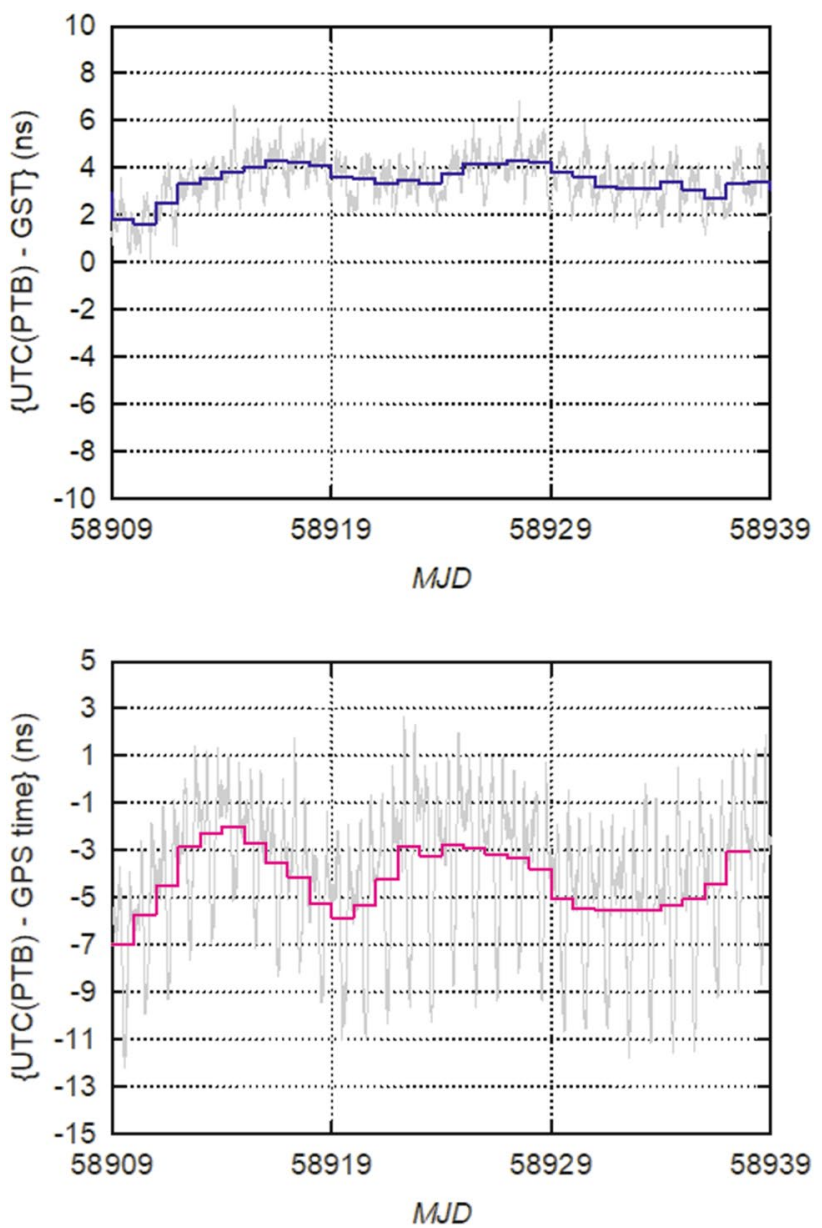

Fig. 9 Reception of Galileo and GPS signals in PTB; here, singlefrequency data collected during the month of March 2020 from a receiver of type MESIT GTR51 are shown. Galileo observations on frequency E1 (upper panel) are corrected for ionospheric delay using the NeQuick model, whereas corrections of GPS observations on frequency L1C rely on the Klobuchar model (lower panel). Gray lines connect mean values of all satellites visible during 16-min intervals, and colored step curves show daily mean values

performed following standard routines to obtain evidence of the performance of the user's local equipment.

The format of the PTB TSB was changed in late 2019 and was presented previously (Piester et al. 2020). What follows is a brief description with reference to two tables that have been reproduced from the first page of a TSB. Explanations to the entries are given in page 2 of each bulletin. The first column in Table 1 of the TSB (here labeled Table 2) lists the Modified Julian Dates (MJD) of the respective week. Column 2 contains information on the German long-wave transmitter DCF77 at $77.5 \mathrm{kHz}$ (Piester et al. 2012) and can be ignored here. The following columns report results of observations of GNSS signal, columns 3 and 4 for GPS, columns 5 and 6 for Galileo. The third column lists daily mean values of the time difference between UTC(PTB) 


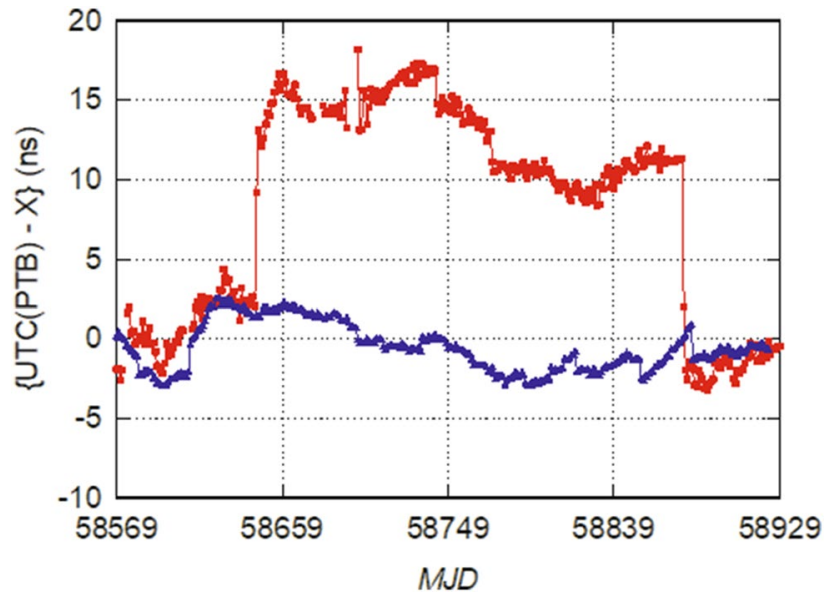

Fig. 10 Comparison of UTC(PTB) with the rapid realization of UTC, UTCr (blue) and with the prediction of UTC as broadcast in the Galileo navigation message (red). The UTCr data are published by BIPM, and the Galileo predictions are reported in RINEX navigation files version 3.02 and higher. A check between RINEX files generated with different software on the receivers from different manufacturers showed perfect agreement. The data span 1 year ending with March 21, 2020 (MJD 58929)

and GPS time (here GPST). The uncertainty of such daily mean values is stated in the explanatory section in page 2 of the bulletin. It is composed of a statistical contribution, which is derived from an analysis of the CGGTTS-formatted data and estimated to be $\mathrm{uA}=1 \mathrm{~ns}(1 \sigma)$. The systematic contribution reflects the status of equipment delay calibration, $\mathrm{uB}=1.5 \mathrm{~ns}$. Column 4 contains the sum of the predicted offset GPST-UTC(USNO), as explained previously, and the measurement results are reported in column 3. Similarly, in column 5 , daily mean values of the time difference between $\mathrm{UTC}(\mathrm{PTB})$ and GST as received are provided. Like column 4, column 6 contains the sum of predicted offset GST-UTC in the Galileo (Gal) navigation message and the measurement results as they are reported in column 5. Column 7 lists the GPS-Galileo Time Offset (GGTO) as contained in the Galileo Signal in Space (SIS) I/NAV Word Type 10 and reported in Galileo navigation files, format RINEX 3.03. The values are reported without an uncertainty stated, and it is not traceable from which satellite observed during the day the information is collected. A substantial offset between this prediction and the measured offset (column 3 minus column 5) like the one reported earlier (see Fig. 10) was reported for many weeks.

In order to facilitate traceability to the international standard UTC, the TSB contains in its second table (here labeled Table 3) data from the most recent Circular T of BIPM. This monthly document is explained in an explanatory supplement referred to in the Circular T. In the table, MJD (column 1) and data from Circular T, Section 1, with combined uncertainty $\mathrm{u}$, in ns, as specified by BIPM (columns 2 and 4) are reported. For comparison, columns 3 and 5 contain the predicted values that had been published in the first table of previous TSB issues, column 6 and column 4, respectively.

Table 2 PTB TSB, report of measurement values (in ns)

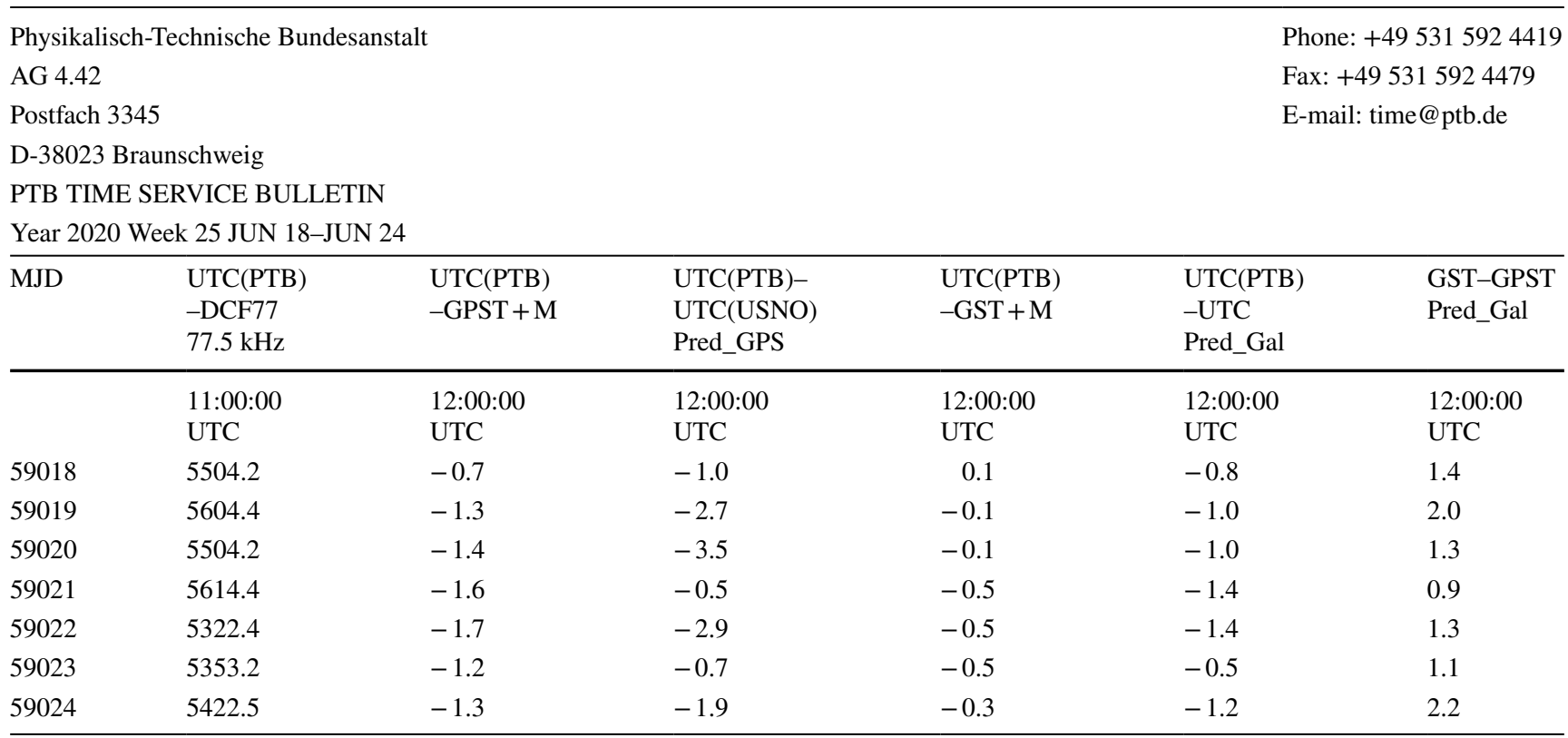


Table 3 PTB TSB, comparison of values from BIPM Circular T with previously reported values

\begin{tabular}{|c|c|c|c|c|}
\hline MJD & $\begin{array}{l}\text { UTC(PTB) } \\
\text {-UTC }\end{array}$ & $\begin{array}{l}\text { UTC(PTB) } \\
\text {-UTC } \\
\text { as pred. }\end{array}$ & $\begin{array}{l}\text { UTC(PTB) } \\
\text {-UTC(USNO) }\end{array}$ & $\begin{array}{l}\text { UTC(PTB) } \\
\text {-UTC(USNO) } \\
\text { as pred. }\end{array}$ \\
\hline & 00:0:00 & 12:00:00 & 00:00:00 & 12:00:00 \\
\hline & UTC & UTC & UTC & UTC \\
\hline & $U=0.6$ & & $U=1.4$ & \\
\hline 58969 & -0.2 & -1.2 & 1.2 & -0.8 \\
\hline 58974 & -0.4 & 0.5 & 1.3 & -0.5 \\
\hline 58979 & -0.5 & 2.3 & 1.2 & -0.3 \\
\hline 58984 & -0.4 & 3.4 & 0.8 & 0.2 \\
\hline 58989 & -0.5 & 1.6 & 0.6 & -0.1 \\
\hline 58994 & -0.8 & 2.1 & -0.4 & -0.9 \\
\hline 58999 & -1.0 & 1.1 & -0.8 & -2.1 \\
\hline
\end{tabular}

\section{Conclusions and summary}

Access to accurate time (in the widest sense) is crucial for many applications in industry and technology. The free availability of GPS signals of excellent quality and reliability has after deactivation of the so-called Selective Availability in 2000 spurred the extensive reliance on GPS as a single source of time and the neglect of other sources. In recent years, a rethinking started, and backup or alternate sources of time get renewed attention. In recent years, requirements for absolute formally unquestionable certainty of time stamps have been codified from European and American financial regulators to the level of $100 \mathrm{~ms}$ (ESMA 2017), and it is likely that in the future, the requirements will be tightened still more. In addition to legal prescriptions or regulations, we see it as a matter of engineering ethics to provide time and frequency measurement results (and time stamps, etc.) based on local references that are traceable to national or international standards and accompanied by a statement of uncertainty. This statement is in line with general metrological requirements. International consistency and comparability of measurements are essential in international collaboration in many application fields as it ensures that measurement results can be universally accepted. This can only be guaranteed if their results are metrologically traceable to internationally recognized references. In our view, this required level of numerical traceability is not attainable by blindly trusting the output of any GNSS device. A project was undertaken by EURAMET, the European Regional Metrology Association, some years ago and ended with the release of a technical guide for calibration laboratories that use GPS-disciplined oscillators as their source of frequency or time traceability to UTC (EURAMET 2016).
At that time, other GNSS were less relevant, but the results would nowadays be applicable for all of them. Considerable variations in regulations across Europe were noted, but there was agreement on a range of core requirements. Calibration of the GNSS-disciplined oscillators is usually recommended if time (epoch) matters in the application and agreement of better than $1 \mu$ s with UTC shall be achieved. Instrumentation should verify correct operation when the device is in use, for example, by monitoring and keeping records of its internal control parameters or by comparing it with an independent second standard. Finally, users are encouraged to make use of the services offered by NMIs and build on the expertise available there.

Acknowledgments We thank the Bundesamt für Kartographie und Geodäsie (BKG) for provision of the equipment of the IGS station PTBB at PTB and Uwe Hessels (BKG) for long-term maintenance and support.

Funding Open Access funding enabled and organized by Projekt DEAL.

Open Access This article is licensed under a Creative Commons Attribution 4.0 International License, which permits use, sharing, adaptation, distribution and reproduction in any medium or format, as long as you give appropriate credit to the original author(s) and the source, provide a link to the Creative Commons licence, and indicate if changes were made. The images or other third party material in this article are included in the article's Creative Commons licence, unless indicated otherwise in a credit line to the material. If material is not included in the article's Creative Commons licence and your intended use is not permitted by statutory regulation or exceeds the permitted use, you will need to obtain permission directly from the copyright holder. To view a copy of this licence, visit http://creativecommons.org/licenses/by/4.0/.

\section{References}

Allan DW, Weiss MA (1980) Accurate time and frequency transfer during common-view of a GPS satellite. In: Proceedings of the 34 th annual frequency control symposium. IEEE, Ft. Monmouth, NJ, pp 334-346

BIPM (2016) BIPM guidelines for GNSS calibration, Version 3.2. https ://www.bipm.org/wg/CCTF/WGGNSS/Allowed/BIPM_guidelines _V3/BIPMcalibration_guidelines_v31.pdf

Bruyninx C, Legrand J, Fabian A, Pottiaux E (2019) GNSS metadata and data validation in the EUREF permanent network. GPS Solut 23:106

Defraigne P, Petit G (2015) CGGTTS-Version 2E: an extended standard for GNSS time transfer. Metrologia 52:G1

Defraigne P, Aerts W, Cerretto G, Cantoni E, Sleewaegen JM (2014) Calibration of Galileo signals for time metrology. IEEE Trans Ultrason Ferroelect Freq Contr 61(12):1967-1975

ESMA (2017) European securities and markets authority (ESMA). Guidelines: transaction reporting, order record keeping and clock synchronization under MiFID II. ESMA/2016/1452, corrected on 7 Aug 2017 
EURAMET (2016) Guidelines on the use of GPS disciplined oscillators for frequency and time traceability. EURAMET Technical Guide No. 3, Version 1.0

EUREF (2020) Permanent GNSS network. http://epncb.oma.be/_netwo rkdata/data_quality/index.php?station=PTBB00GER

European Commission (2015) European GNSS (Galileo) open service: ionospheric correction algorithm for galileo single-frequency users. https://doi.org/10.2873/685913

Feldmann T, Bauch A, Piester D, Rost M, Goldberg E, Mitchell S, Fonville B (2010) Advanced GPS-based time link calibration with PTB's new GPS calibration setup. In: Proceedings 42nd annual precise time and time interval (PTTI) systems and applications meeting, Reston, Virginia, USA, November 15-18, pp 509-526

Galluzzo G, Lucas Rodriguez R, Morgan-Owen S, Binda S, Blonski D, Crosta F, Gonzales F, Molina Garcia J, Otero X, Sirikan N, Spangenberg M, Spinelli E, Swinden R, Wallner S (2018) Galileo system status, performance metrics and results. In: Proceedings of ION ITM 2018. Institute of Navigation, Reston, Virginia, January 29-February 1, pp 790-809

Garbin E, Defraigne P, Krystek P, Píriz R, Bertrand B, Waller P (2019) Absolute calibration of GNSS timing stations and its applicability to real signals. Metrologia 56(1):015010

GPS (2019) Global positioning systems directorate, Navstar GPS space segment/navigation user interfaces IS-GPS-200 Rev. K. https:// www.navcen.uscg.gov/?pageName $=$ gpsTechnicalReferences

GPS SPSPS (2020) Global positioning system standard positioning service performance standard, 5th edn. April 2020. https://www. gps.gov/technical/ps/2020-SPS-performance-standard.pdf

IGS (International GNSS Service) (2013) IGS site guidelines, infrastructure committee. IGS Central Bureau. https://kb.igs.org/hc/ en-us/articles/202011433-Current-IGS-Site-Guidelines

IGS (International GNSS Service) (2015) The receiver independent format exchange RINEX. Working group and radio technical commission for maritime services special committee 104 (RTCMSC104). ftp://igs.org/pub/data/format/rinex303.pdf

ITU (International Telecommunication Union) (2010) Handbook satellite time and frequency transfer and dissemination, Geneva, Switzerland

JCGM (Joint Committee for Guides in Metrology) (2012) International vocabulary of metrology - basic and general concepts and associated terms (VIM), 3rd edn. JCGM 200:2012

Jiang Z, Arias F, Lewandowski W, Petit G (2011) BIPM calibration scheme for UTC time links. In: Proceedings of 2011 joint conference of the IEEE international frequency control symposium the european frequency and time forum, May 02-05, San Francisco, USA, pp 1064-1069

Johnston G, Riddell A, Hausler G (2017) The international GNSS service. In: Teunissen, Peter JG, Montenbruck O (eds) Springer, handbook of global navigation satellite systems, 1st edn. Springer International Publishing, Cham, pp 967-982

Lombardi M (2016) Evaluating the frequency and time uncertainty of GPS disciplined oscillators and clocks. NCSLI Meas 11(3-4):30-44

Matsakis J, Levine J, Lombardi MA (2018) Metrological and legal traceability of time signals. In: Proceedings ION PTTI 2018. Institute of Navigation, Reston, Virginia, January 29-February 1, pp 59-71

Panfilo G, Arias F (2019) The coordinated universal time (UTC). Metrologia 56(4):042001

Petit G, Harmegnies A (2019) Tests of Galileo and BeiDou links for UTC. In: Proceedings 2019 joint conference of the ieee international frequency control symposium the european frequency and time forum, April 14-18, Orlando, USA, pp 34-36

Piester D, Bauch A, Becker J, Hoppmann A (2012) Time and frequency broadcast with DCF77. In: Proceedings of 43rd annual precise time and time interval (PTTI) systems and applications meeting, 14-17 Nov 2011, Long Beach, California, USA, pp 185-196

Piester D, Bauch A, Polewka T, Staliuniene E, Teichel K (2019a) Disciplined oscillators for traceable frequency and time in metrology and financial sectors. Navigation 66:661-671

Piester D, Bauch A, Peik E, Polewka T, Staliuniene E, Teichel K (2019b) An uncertainty study on traceable frequency and time with disciplined oscillators for metrology and financial sectors. In: Proceedings of 2019 joint conference of the IEEE international frequency control symposium and European frequency and time forum (IFCS-EFTF 2019), Orlando, Florida, USA, April 14-18

Piester D, Bauch A, Becker J, Gutbrod M, Klein T, Polewka T, Sibold D, Staliuniene E, Teichel K, Vajen W (2020) PTB's time and frequency services 2018-2019. Proceedings ION 2020 precise time and time interval systems and applications meeting, January 21-24. San Diego, California, pp 146-158

Píriz R et al (2015a) The time validation facility (TVF): an all-new key element of the galileo operational phase. In: Proceedings of 2015 joint conference of the IEEE international frequency control symposium the European frequency and time forum, April 12-16, Denver, USA, pp 320-325

Píriz R, Rodriguez D, Roldan P, Mudrak A, Bauch A, Leute J, Pánek P, Kuna A (2015b) Relative calibration of galileo receivers within the time validation facility (TVF). In: Proceedings of 2015 joint conference of the IEEE international frequency control symposium \& European frequency and time forum, Apr 12-16, Denver, Colorado, USA, pp 245-249

Píriz R, Roldán P, Golcz R, Moriana GC, Leute J (2016) Performance of the NeQuick G Iono model for single-frequency GNSS timing applications. In: Proceedings of the European frequency and time forum 2016 (EFTF 2016) April 4-7, York, UK, pp 144-147

White J, Beard R, Landis G, Petit G, Powers E (2001) Dual frequency absolute calibration of a geodetic GPS receiver for time transfer. In: Proceedings of 15th European frequency and time forum, March 6-8, Neuchâtel, Switzerland, pp 167-170

Publisher's Note Springer Nature remains neutral with regard to jurisdictional claims in published maps and institutional affiliations.

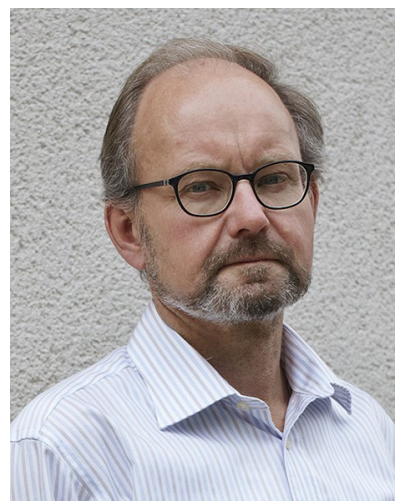

Andreas Bauch has a Ph.D. in physics and joined PTB in 1983. He has always been involved in time and frequency metrology, focused at first on the development and operation of atomic clocks, later more and more on time comparison techniques (GNSS, TWSTFT). Today he is Head of PTB's Time Dissemination Working Group and, as such, the management responsible for the operation of PTB's time dissemination services. 


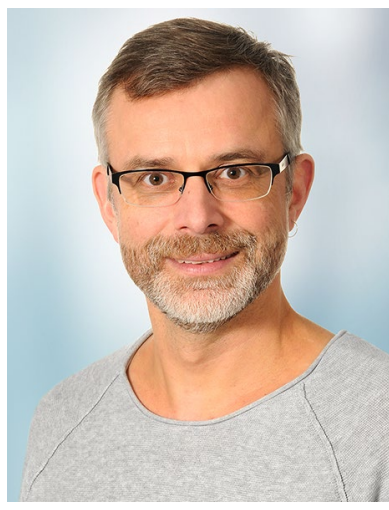

Dirk Piester joined the Time Dissemination Group of PTB in 2002 where he is involved in maintaining and development of PTB's time services and international clock comparisons supporting the production of UTC. His research activities include time and frequency metrology via telecommunications and navigation satellites, as well as fiber-optic communication. Also, he is engaged in technical assessments for Germany's national accreditation body DAkkS.
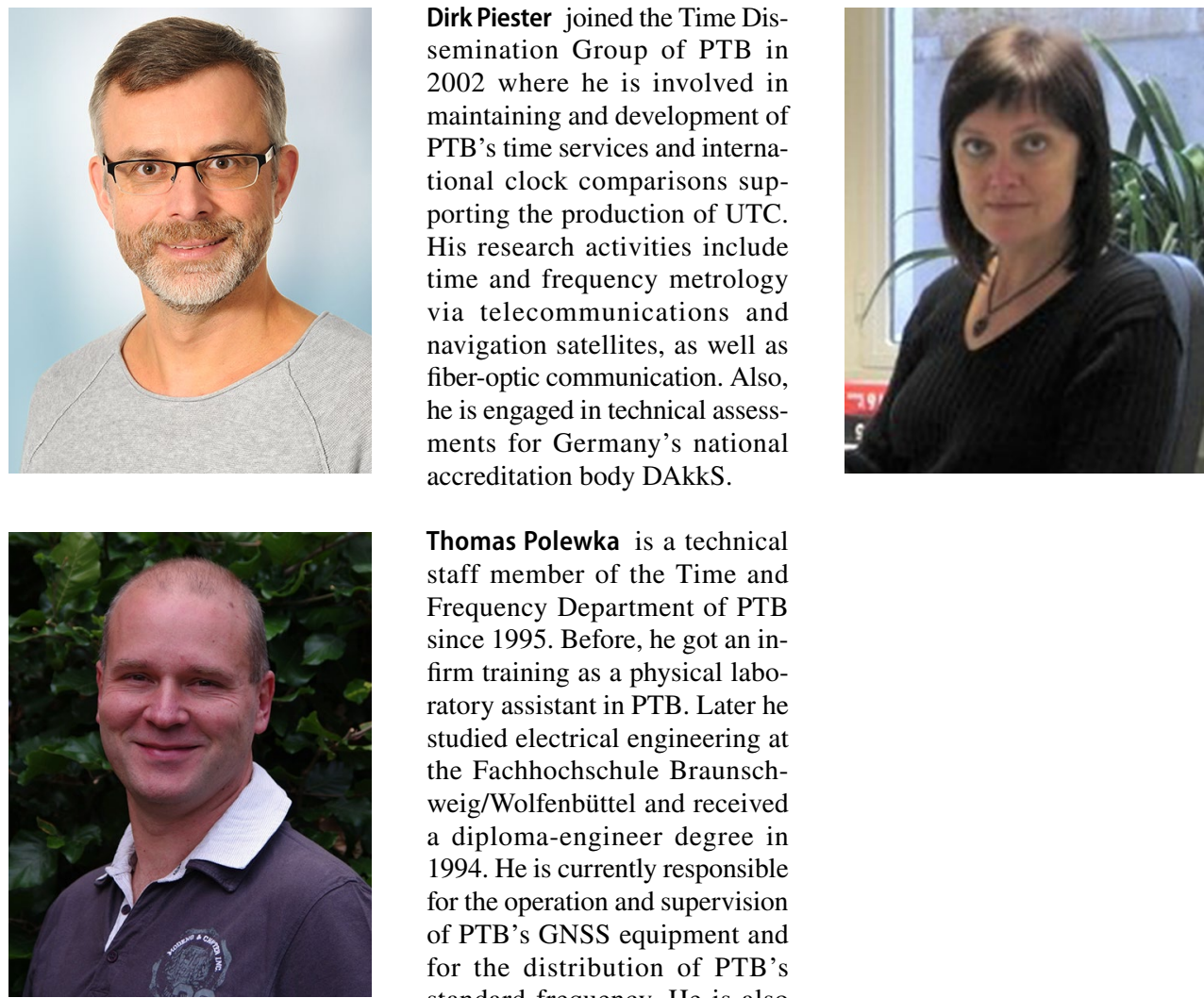

Thomas Polewka is a technical staff member of the Time and Frequency Department of PTB since 1995. Before, he got an infirm training as a physical laboratory assistant in PTB. Later he studied electrical engineering at the Fachhochschule Braunschweig/Wolfenbüttel and received a diploma-engineer degree in 1994. He is currently responsible for the operation and supervision of PTB's GNSS equipment and for the distribution of PTB's standard frequency. He is also engaged in technical assessments for Germany's national accreditation body DAkkS.
Egle Staliuniene received her master's degree in mathematical modeling and cybernetics from the Vilnius University, Lithuania, with the thesis "Application of adaptive statistical models for short time range forecasting of economic data series." Since 2000 , she is with PTB as a technical assistant and programmer. In 2003, she joined PTB's Time Dissemination Group and is responsible for IT solutions of process automatization and control, data transfer, processing and visual representation. 